Nouvelles perspectives en sciences sociales

Revue internationale de systémique complexe et d'études relationnelles

\title{
Pour une sociologie de l'écart. Affiliation et différenciation dans les processus de socialisation et d'urbanisation
}

\section{Franck Dorso}

Volume 8, numéro 1, novembre 2012

URI : https://id.erudit.org/iderudit/1013917ar

DOI : https://doi.org/10.7202/1013917ar

Aller au sommaire du numéro

Éditeur(s)

Prise de parole

ISSN

1712-8307 (imprimé)

1918-7475 (numérique)

Découvrir la revue

Citer cet article

Dorso, F. (2012). Pour une sociologie de l'écart. Affiliation et différenciation dans les processus de socialisation et d'urbanisation. Nouvelles perspectives en sciences sociales, 8(1), 35-59. https://doi.org/10.7202/1013917ar

\section{Résumé de l'article}

Le texte aborde la question de la tension entre homogénéisation et différenciation à partir des usages informels et illégaux de la ville. Au-delà des condamnations dont ils font l'objet, ces usages remplissent des rôles essentiels de régulation, au Nord comme au Sud, en termes économiques, urbains, sociaux, politiques. Ils constituent des formes de soupape des processus d'urbanisation mais aussi, plus globalement, de socialisation. Ils permettent dans ce registre des épisodes de retrait ou de transgression vis-à-vis du contexte normatif, sans pour autant remettre en cause l'ordre social. Cette logique de l'écart renvoie à la tension affiliation normative-différenciation qui innerve la socialisation. Le texte explore la dynamique de cette tension, comme une modalité du couple homogénéisation-différenciation, ainsi que ses implications en termes sociologiques et d'action urbanistique. 


\title{
Pour une sociologie de l'écart. Affiliation et différenciation dans les processus de socialisation et d'urbanisation
}

\author{
Franck Dorso \\ Université Paris-Est Créteil Val de Marne, \\ Institut d'urbanisme de Paris
}

\section{Introduction}

Tes pratiques illégales et les usages informels de la ville sont U fréquemment considérés comme des différences perturbatrices et des désordres. Pourtant, certains de ces processus peuvent être vus comme des éléments indispensables de l'ordre urbain, des garants de sa continuité - sous des formes variées selon les contextes mais partageant des logiques similaires. L'écart, les écarts, fonctionnent comme des soupapes nécessaires à la vie urbaine et plus globalement sociale, jusqu'au processus de socialisation des individus. La problématique de la différenciation sera abordée ici à partir des logiques de l'écart identifiées dans des recherches en cours sur plusieurs terrains.

Certains usages informels, illégaux ou transgressifs renvoient à des moments de résolution d'une tension structurante dans le processus de socialisation, entre affiliation normative et différenciation. Les individus expriment le besoin de se retirer ou de transgresser les normes du quotidien, soit pour " souffler " 
(retrait), soit pour s'affirmer, se construire, exprimer une singularité (transgression). Dans les formes étudiées, ce plan est distinct des mobilisations à visée transformatrice de la société : une fois l'écart réalisé, les individus souhaitent réintégrer le cours normal de leur vie - comme une sortie de piste très temporaire, mais dont la forme est marquante et peut poser problème dans la police de la cité. Les acteurs peuvent avoir une conscience diffuse de ce mécanisme de régulation informelle, mais celui-ci doit rester le plus souvent dans le non-dit, voire la dénégation, pour pouvoir jouer son rôle régulateur.

Le texte commencera par présenter les résultats d'enquêtes de terrain qui ont mené aux logiques de l'écart et la dynamique de ce processus. Puis, à partir d'un certain nombre de paradoxes posés en termes d'analyse comme dans le champ de l'action, il proposera quelques pistes pour avancer dans la proposition d'un projet de recherche centré sur la problématique de l'écart.

\section{Les logiques de l'écart}

\subsection{Résistances de l'informel}

Le terme d'informel recouvre plusieurs situations et reste sujet à discussion, selon le type d'activité et de contexte ${ }^{1}$. Jusqu'au mot lui-même : on ne peut pas dire en effet que l' « informel " manque de forme, dans la mesure où ce sont le plus souvent ses matérialisations dans les espaces vécus qui s'érigent en problèmes à résoudre ${ }^{2}$. On désigne en fait le plus souvent a minima sous le terme d'informel des activités illégales, c'est-à-dire qui se déroulent et se déploient sans autorisation ou en violation des règles ou des usages établis. C'est le cas par exemple des deux formes les plus visibles - et qui ont fait l'objet des travaux les plus importants : l'habitat illégal (quartiers clandestins, invasions,

1 Michel Agier, L'Invention de la ville. Banlieues, townships, invasions et favelas, Amsterdam, Éditions des archives contemporaines, 1999. Jérôme Monnet, " Dissociation et imbrication du formel et de l'informel : une matrice coloniale américaine ", Espaces et sociétés, 2010, vol. 3, n 143, 2010, p. 13-29.

2 Franck Dorso, "Informel ", dans L'Abc de l'urbanisme, Créteil, Université Paris-Est Créteil éditeur, 2010, p. 91-94. 
favelas, gecekondu...) et l'économie non déclarée (travail au noir, commerce de rue, activités économiques illicites...).

À ces deux types s'en ajoute un troisième, lié aux usages plus éphémères, qui n'impliquent pas une aussi grande stabilité que l'habitat et l'économie : trajets, parcours sur des zones interdites, libations, sexualité, isolement, repos, marquages de l'espace... Toutes ces pratiques ne représentent pas forcément des délits, mais sont considérées comme des risques, ou des outrages aux bonnes mœurs, et se déploient dans des espaces-temps cachés ou discrets. Ce type d'usages informels est moins immédiatement visible. Ses traces sont plus légères, et s'inscrivent dans des temporalités plus courtes ou mouvantes (un même lieu peut être occupé normalement puis plus étrangement selon les moments de la journée, mais aussi de l'année selon les saisons, les événements, etc.). Il est aussi moins étudié dans les recherches sur l'informalité. Il est pourtant très répandu, et n'est pas réservé aux contextes d'urbanisation rapide : on le trouve partout, aux Nords comme aux Suds.

Ces trois formes sont présentes sur les différents terrains abordés depuis une dizaine d'années en France (Tours, Rennes, Île de France) et en Turquie (Istanbul). Je m'appuierai dans ce texte sur le terrain Turc. Il a ouvert le premier sur les logiques de l'écart, et les enquêtes de terrain réalisées cette année ont permis les développements présentés ici.

À Istanbul, les occupations illégales du site de la muraille antique qui entoure la ville historique se sont développées à partir du XIX ${ }^{e}$ siècle, lorsque l'édifice a progressivement perdu son utilité militaire : d'abord avec l'artisanat et les activités agricoles (pâturage, maraîchage), puis, à partir des phases d'expansion urbaine dues à l'exode rural dans les années 1950 puis 1970, avec l'habitat de fortune, progressivement "durcifié ", à l'intérieur des trois rangées de murs, dans les tours, et devant les remparts. Cette colonisation du site a favorisé le développement du troisième type de pratiques informelles éphémères, et celles-ci sont fort nombreuses sur les sept kilomètres de long de la partie terrestre de la muraille de Théodose II. En 1985, la ville d'Istanbul 
est inscrite sur la liste du patrimoine mondial de l'UNESCO. Commence alors une phase de conflit larvé entre les autorités qui sont sommées de dégager le site pour procéder à des travaux de conservation sur le rempart, et les occupants et utilisateurs présents sur place. Presque trente ans plus tard, les usagers informels parviennent à maintenir leur présence sur les lieux, même si celle-ci a pu évoluer sur certaines portions : moins d'habitat (en fait celui qui était visible depuis les rues à l'extérieur), moins d'activités économiques, mais autant sinon plus d'activités éphémères.

Cette recherche sur Istanbul s'inscrit dans plusieurs étapes. Après deux phases en 2000-2001 puis en 2005-2008, une troisième phase a démarré en 2011. Ces travaux ont mobilisé les outils de l'enquête sociologique et anthropologique, en articulant le recueil de matériaux auprès des institutions (entretiens, documents), la participation à des événements (cérémonies de commémorations historiques, expulsions, chantiers) et les enquêtes sur le terrain, auprès des usagers. Lors de ces enquêtes, plusieurs outils ont été utilisés : entretiens in situ, questionnaires (qui ont rapidement évolué vers des formes adaptées aux contraintes de terrain), cartes mentales et identifications photographiques, observations postées. Mais surtout j'ai privilégié l'immersion sur le terrain et sur la durée, en pratiquant l'observation participante, en vivant sur les sites, en menant différentes pratiques, parfois en endossant des rôles (avec les interrogations déontologiques qui accompagnent ces actes ${ }^{3}$ ) pour avoir accès à des groupes, des sites ou des pratiques plus difficiles à pénétrer.

Sur différents terrains abordés, en Turquie comme en France, les usages informels se poursuivent sur les lieux en dépit de condamnations publiques et d'opérations de déguerpissement ou de dégagement menées périodiquement. Par ailleurs, les acteurs de l'informel ne se mobilisent pas collectivement, face à des autorités locales qui, au moins dans les discours, et parfois dans

Franck Dorso, La Part d'ombre. Transactions et conflits entre les usages informels et les opérations de rénovation de la muraille de Théodose II à Istanbul, mémoire de thèse, Strasbourg, CRESS EA 1334, Université Marc Bloch, 2008. 
les actes, montrent qu'elles ont les moyens de réduire ces occupations. La problématique de départ de ces recherches portait sur ce rapport de force inversé : comment les usagers informels maintiennent-ils leur emprise et leurs usages dans des contextes aussi défavorables en regard du rapport de force objectif vis-à-vis des autorités ? C'est en répondant à cette question que la recherche a évolué, ouvrant, entre autres, la question de la tension entre différenciation et affiliation normative.

\subsection{Désordre urbain et régulation}

À Istanbul, les pratiques informelles jouent un rôle de régulation au niveau du processus d'urbanisation, mais aussi, comme on le verra, dans le processus de socialisation des individus. Les pratiques autour de l'habitat et de l'économie offrent des solutions en l'absence de politiques publiques adéquates ou de ressources propres des usagers. L'expansion urbaine de la ville se poursuit à un rythme élevé, et le contexte socio-économique continue de produire des fragilités. Loin des taux de croissance affichés de l'économie turque, les habitants des quartiers populaires restent soumis à la précarité de l'emploi, et à celle de leur logement, dans le cadre d'un renouvellement urbain qui ouvre des chantiers profitables mais peut déplacer des populations ou des activités économiques sans ménagement ${ }^{4}$. Le secteur de la construction continue d'être le fleuron de l'économie stambouliotes. Même si le logement clandestin a reculé sous l'effet d'une réorganisation du secteur de la construction, il peut perdurer sous des formes très précaires. Mais c'est surtout sur le plan de l'activité économique que l'activité informelle peut se révéler indispensable : les périodes de chômage, les faibles salaires, la rareté des contrats fixes nécessite fréquemment d'avoir recours à une activité non déclarée ou, souvent, à cumuler deux ou trois emplois, dont un seul sera légal.

$4 \quad$ Muriel Girard, Recomposition du monde artisanal et mutations urbaines au regard des mises en patrimoine et en tourisme au Maghreb et au Moyen-Orient (Fès, Istanbul, Alep), mémoire de thèse, Tours, UMR 1835 CITERES, Université François Rabelais, 2010.

5 Jean-François Pérouse, La Turquie en marche, Paris, La Martinière, 2004. 
Ces activités informelles permettent ainsi de résoudre plusieurs tensions sur les plans urbain, économique, social. Les acteurs concernés, qu'ils soient usagers de terrain, représentants des autorités ou acteurs économiques influents, ont une conscience diffuse de ce rôle pratique de "soupape ". Certes les réponses institutionnelles et légales peuvent diverger selon les contextes, allant d'une acceptation implicite à des mesures radicales de " nettoyage ", en passant par un gradient d'attitudes intermédiaires, parfois ambiguës. À Istanbul, l'intérêt objectif pour ces soupapes à moindres frais explique en partie leur continuité, autour d'arrangements et de transactions subtils, qui doivent rester tacite pour fonctionner, et dans lesquels les acteurs n'ont pas toujours une compréhension totale de la situation.

Cette "in-connaissance " est justement perçue comme une condition nécessaire à l'établissement de ces compromis : si l'on ne sait pas tout, il reste juste de ne pas tout maîtriser. Il ne s'agit pas tant d'une méconnaissance, car chacun sait un peu ce qui se passe sur le terrain, mais d'une réserve volontaire pour ne pas atteindre un niveau de connaissance qui ne permettrait plus de ne pas agir, sans se mettre dans une position ouvertement illégitime et risquée. Cette ignorance travaillée est un enjeu pour garantir le non-dit qui arrange tout le monde à un moment donné. J'ai pu faire ce constat dans les entretiens auprès des acteurs de la municipalité à Istanbul, ainsi que chez certains experts de la conservation architecturale, mais parfois aussi en France, sur un registre assez proche. Ce jeu d'intérêts et de compromis fonctionne bien souvent comme un élément stabilisateur et pacificateur, autour de tolérances concrètes.

Ce mécanisme de soupape œuvre également dans le troisième registre des usages informels plus labiles. Il révèle un processus qui dépasse, dans ce cas, les registres urbains et économiques pour toucher au processus de socialisation des individus. En effet, ce qui motive ces actes éphémères ne trouve pas seulement son origine dans des tensions liées aux transformations urbaines ou à la situation économique, mais, de façon permanente, dans la 
vie sociale des individus, dans leur rapport quotidien aux autres et au monde social.

J'utilise pour le moment le terme de logiques de l'écart pour décrire ce processus, et le propos sera donc centré à partir de maintenant sur les usages éphémères diffus et omniprésents qui révèlent ce processus. Il est possible que la mécanique de l'écart fonctionne avec des similitudes dans les trois types d'informalités, puisqu'il s'agit dans tous les cas de mécanismes de régulation, mais elle s'incarne pour les trois cas dans des registres (et ouvre donc des perspectives analytiques) différents.

\subsection{Les pratiques de l'écart}

Les abords de l'Edirnekap1, au point culminant de la muraille terrestre d'Istanbul, ont lentement changé en dix ans. Les travaux de rénovation qui ont suivi le séisme d'août 1999 ont relevé certaines tours, et reconstruit à la hâte et sans vrai relevé archéologique des portions disparates des deux murs principaux. Mais ce sont surtout les chantiers environnants qui ont amené des modifications : la reconstruction très controversée de l'ancien quartier Rom de Sulukule, côté intérieur, et le prolongement d'une ligne de tramway côté extérieur sont en train d'avoir raison des derniers habitats aménagés dans les niches et les tours. Pour autant, les usages éphémères des lieux n'ont pas beaucoup changé. Quand la municipalité a songé, à partir de 2006, à céder par baux emphytéotiques à des promoteurs amis quelques portions de mur pour une exploitation touristique et hôtelière, des grilles ont été installées, mais leurs serrures ont disparu en même temps que l'appétit des développeurs. Si la prostitution organisée plus bas vers la trouée d'Ulubatlı semble avoir disparu, les errances et les libations nocturnes restent quant à elles bien en place. Pendant la journée, de nombreuses personnes gagnent les parties herbeuses ou grimpent sur les remparts pour se reposer, dormir, s' isoler un moment. D'autres vont manger, voire improvisent un mangal, caisse de fer léger accueillant un petit barbecue. Des groupes discutent aussi, assis à l'ombre des quelques arbres qui ont poussé devant l'ancien rempart. 
Les parties rénovées sont censées accueillir librement les visiteurs, mais la muraille de Théodose II est un espace appartenant à la municipalité, un site historique livré temporairement à des chantiers de réparations, et dont l'usage doit se limiter à la visite, le plus souvent à l'extérieur des murs eux-mêmes. Dans les faits, les touristes sont très rares, alors que les appropriations spontanées, pourtant interdites, sont nombreuses, ce que la municipalité et les médias ne manquent pas de condamner. Les maraîchers installés dans l'ancien fossé comblé le long de la partie sud constituent un cas à part, assez ambigu, autour d'une location aux contours fragiles et soumis à une grande précarité.

Cependant, la première condamnation des usages vient des riverains des quartiers populaires voisins : nombre d'habitants déplorent les usages du site, non seulement pour leurs conséquences matérielles, mais surtout pour leur caractère d'outrage aux bonnes mœurs. Les libations alcoolisées sont bien sûr condamnées, mais aussi les toxicomanies, et l'errance de "paumés » qui, aux dires des habitants, sont forcément des chômeurs : la muraille est présentée explicitement comme un coupe-gorge où « tu dois te méfier de pas prendre un coup de couteau, n’y vas pas!"

Maintes pratiques réprouvées viennent en effet s'abriter dans la muraille. On reste toutefois assez loin du coupe-gorge annoncé. De nombreux rituels de co-présence assurent une relative sécurité des lieux. Les usagers, les premiers, ont intérêt à cette pacification endogène du site, pour pouvoir l'utiliser dans de bonnes conditions. L'image de bas-fond ne correspond pas à la réalité, et les usagers de la muraille ne sont pas majoritairement des exclus ou des gens en difficulté - même s'il y en a, notamment dans les habitats. Il m'est arrivé de rencontrer en pleine action sur la muraille des personnes qui, dans leur quartier, à l'ombre des hauts murs proches, pouvaient se faire les censeurs virulents de son usage. Il y a dans ce décalage et ces doubles jeux la construction d'une figure bien commode du repoussoir, qui joue un rôle important dans la construction normative : une image négative nécessaire en regard de laquelle peuvent s'ériger les normes de la 
bonne ville, des bons comportements et des bons usages, comme une modalité de la tension identité-altérité par laquelle on commence par définir ce que l'on est en désignant ce que l'on n'est pas, ou ne doit pas être. L'image du coupe-gorge fonctionne ici comme une prescription.

Lors des enquêtes réalisées auprès des usagers éphémères, les enquêtés prétendent "souffler", venir chercher de la tranquillité. L'observation participante est ici fort utile, car certains arguments ne donnent pas lieu à de grands épanchements, parfois seules les attitudes parlent : on vient se retirer ici pour faire une sieste ou parler ou boire entre amis sans être embêté. Une part des usages éphémères répondent ainsi à un besoin d'échapper quelques instants à la pression sociale, morale et normative des espaces publics ou familiers.

À côté de ces pratiques plutôt calmes, d'autres usages ont, on l'a vu, un caractère moins tranquille : les réunions nocturnes, les marquages, certaines pratiques festives ou sexuelles. On peut ainsi distinguer deux catégories de pratiques éphémères : des pratiques relevant du retrait, d'autres relevant de la transgression.

Il n'y a pas dans ces pratiques une volonté de changer le monde ou de changer sa vie. Les usagers souhaitent retourner à leurs rôles sociaux classiques après avoir quitté cette scène secondaire. Ils quittent pour un instant la scène normée quotidienne de la rue pour passer sur des scènes différentes, une sorte de coulisse de la ville officielle, pour reprendre l'expression d'Erwin Goffman ${ }^{6}$. Bien sûr, les normes ne s'abolissent pas sur ces scènes secondaires, en dépit des discours sur le relâchement de la pression. Simplement elles diffèrent. On passe donc d'un contexte normatif à un autre, mais le ressenti de cette différence est la clef du passage à l'acte. Le terme de "pratiques de l'écart " désigne ces retrait et transgression qui permettent de ressentir un écart à la norme et au quotidien.

Cette dynamique semble renvoyer à un moment particulier du processus de socialisation. La construction de soi s'opère dans

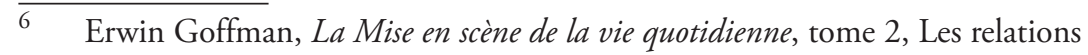
en public, Paris, Minuit, 1973. 
un mouvement d'affiliation aux normes, dans le besoin de confiance, de reconnaissance, de cadres et de routines. Mais cette affiliation impose des pressions fortes, à travers des contraintes qui sont ressenties plus ou moins consciemment sur les désirs, les pulsions, les comportements, les actes et les représentations. Cette pression est rendue vivable par la possibilité d'épisodes de décompressions, qui permettent de se relâcher, mais aussi de transgresser les interdits, de se mettre à l'épreuve, de ressentir son individualité et sa singularité. Ces épisodes peuvent être plus vifs et prégnants à certaines étapes de la vie (petite enfance, adolescence, changements dans les parcours de vie), mais la tension est présente tout le temps. Aussi de petites et fréquentes décompressions semblent intervenir régulièrement. Elles prennent plusieurs formes, et les pratiques de retrait et de transgressions en sont une expression.

\subsection{Affiliation - différenciation}

Une tension structurante dans le processus de socialisation des individus se dessine, caractérisée par deux pôles : affiliation normative versus différenciation. Ces deux forces structurent la socialisation au long cours des individus. Engagées sur la question du rapport de force et à partir de références bourdieusiennes, ces recherches ont vu le cadre théorique évoluer. L'identification de la tension structurante est liée à l'utilisation conjointe du paradigme de la transaction sociale, initié par Jean Rémy et Liliane Voyé ${ }^{7}$ L'intérêt de ce paradigme réside dans la préférence pour une approche cumulative plutôt qu'exclusive dans les appuis théoriques $^{8}$, ce qui permettait de ne pas abandonner les autres points de vue, et de tenter de les articuler.

C'est un point important, car l'exploration des logiques de l'écart - leur caractère, semble-t-il, étendu dans la vie sociale et

7 Jean Rémy et Liliane Voyé, Ville, ordre et violence, Paris, Presses universitaires de France, 1981; Jean Rémy, Liliane Voyé et Émile Servais, Produire ou reproduire, tome 1, Conflits et transactions sociales, Bruxelles, Vie ouvrière, 1978.

8 Jean Rémy, Sociologie urbaine et rurale. L'espace et l'agir, Paris, L'Harmattan, 1998. 
les différents groupes sociaux - ne doit pas faire oublier les contraintes (de position, de ressources) qui pèsent sur chacun. Tous les individus ne vont pas vivre les mêmes objectivations dans la résolution de la tension. Sur les terrains étudiés, j’accorde de l'importance à la spatialisation de cette objectivation et je rencontre certains types de pratiques et de groupes. Mais toutes les résolutions de la tension affiliation-différenciation n'empruntent pas les mêmes voies ni les mêmes formes, spatiales ou temporelles. Dans les groupes, par exemple fort pourvus en capital économique, il est sans doute plus facile d'aller se retirer ou transgresser dans des situations plus légales (mobilités de loisir lointaines, vies parallèles) que de descendre dans le terrain vague voisin.

Dans l'approche en termes de transaction sociale, les situations rassemblent les acteurs autour d'enjeux pratiques et sont caractérisées par une triple relation : échange, négociation et rapport de force. Ces relations donnent une marge de manœuvre aux acteurs impliqués, mais ceux-ci restent contraints, d'une part, par ce qui échappe à leur intentionnalité (les déterminants objectifs de leurs positions, qui sont à la fois le contexte général mais aussi leurs propres valeurs); d'autre part, par une dimension structurale propre à la situation elle-même. Dans la lignée de Simmel ${ }^{9}$ puis de Ledrut ${ }^{10}$, le paradigme reprend l'idée que les situations sont structurées par des couples d'opposés à la fois inséparables et inconciliables. Il n'existe pas de solution définitive ou durable à la tension, et pourtant la vie continue. Dans les faits, des résolutions temporaires interviennent, sous la forme de compromis pratiques et transitoires, qui, par les dynamiques de l'échange, de la négociation et du rapport de force, permettent de résoudre pour un temps le conflit ${ }^{11}$.

9 Georg Simmel, Sociologie et épistémologie, Paris, Presses universitaires de France, coll. "Sociologies", 1989.

10 Raymond Ledrut, L'Espace en question, Paris, Anthropos, 1976.

11 Maurice Blanc, "La Transaction, un processus de production et d'apprentissage du "vivre-ensemble" ", dans Maurice Blanc, Marie-France Freynet et Gérard Pineau (dir), Les Transactions aux frontières du social, Lyon, Chronique Sociale, 1998, p. 219-238. 
La tension et l'opposition ne disparaissent pas dans ces compromis, et au bout d'un certain temps, la tension redevient suffisamment vive pour nécessiter l'établissement d'un nouveau compromis. Ce mouvement montre ainsi une succession de séquences transactionnelles, dans lesquelles vient prendre place le changement social, à travers l'évolution plus ou moins marquée de certains éléments du contexte : changement des acteurs impliqués ou reconnus, changement des ressources qui structurent les rapports de positions, changement des éléments matériels (lois, légitimités, objets, temps, espaces).

Sur le terrain, les usages informels peuvent être analysés dans le cadre d'une double transaction : une transaction visible sur l'appropriation d'espaces-temps, et une transaction plus profonde sur la régulation sociale et normative. Bien sûr, ces deux plans sont reliés dans les faits : les pratiques de l'écart permettent de résoudre temporairement une tension structurante du processus de socialisation, et cette résolution affecte, en aval, la vie urbaine, le processus d'urbanisation, la fabrique de la ville. Il y a donc aussi deux niveaux de compromis : un compromis interne à la tension structurante (la pratique de l'écart comme résolution temporaire de la tension structurante du processus de socialisation), et un compromis externe ou objectivé qui doit s'établir dans l'attitude des autorités vis-à-vis de ces usages illégitimes mais potentiellement intéressants pour conserver une certaine paix sociale.

\section{2. Étapes pour une analyse de l'écart}

L'écart se présente comme une différence ambiguë : une opposition radicale au contexte normatif mais essentielle à sa régulation; des formes condamnées par les autorités mais utiles à la conservation de l'ordre. Je voudrais présenter maintenant les pistes de travail qui s'ouvrent sur la question de l'écart et de la différenciation, comme une manière aussi de les mettre en débat. 


\subsection{Retrait et transgression}

Sur le terrain, les usages informels éphémères se répartissent en deux catégories : certains semblent plus actifs et marqués par la transgression, d'autres apparaissent plus passifs, comme les pratiques d'isolement, de déambulations, de repos, de repas, plus souvent solitaires, ou en très petit groupe.

C'est le cas des très nombreuses présences discrètes tout le long de la muraille d'Istanbul. Je discutais un jour avec un homme assis tranquillement sur un promontoire près de Topkapı (une porte de la muraille terrestre, et non le palais ottoman) en fin d'après-midi. Il me confia être un policier qui venait souffler ici en fin de service avant de rentrer chez lui. Comme un sas de décompression entre deux sphères principales d'engagement, le travail et la famille. Cet échange est caractéristique des entretiens in situ que j'ai pu faire en journée sur les terrains, en Turquie comme en France. Plus loin au sud, près de Yedikule (le château des sept tours), plusieurs interstices entre les tours ou sur le sommet de tours arasées par l'usure abritent des repas, tranquilles, solitaires, en couple ou en petits groupes. Dans certains cas, un foyer est hâtivement dressé. Ces appropriations restent momentanées, et leur ressemblance avec les habitats clandestins est trompeuse. Dans ce dernier cas, en effet, des marques d'occupation sont disséminées aux abords du campement (linge, ustensiles) pour signifier l'appropriation, tout le monde comprend ces signes et contourne les abords immédiats, dans des rites d'usage et de co-présence assez établis. Ici ce n'est pas le cas, il s'agit le plus souvent d'habitants de quartiers proches, ou de personnes qui travaillent à proximité, et qui se mettent de côté un moment. Cette pratique du repas se retrouve en maints endroits de la muraille, dans les lieux qui offrent une discrétion relative, sans aller toutefois dans les espaces les plus cachés.

Plusieurs relevés d'occupation, à travers notamment des observations postées en un même lieu avec comptage et grilles d'observation, ont révélé que des personnes faisaient de courtes haltes sur la muraille, pour se reposer, pleurer, marcher seules, ou plus longuement faire une sieste ou manger, avant de reprendre 
leur chemin. C'est le cas en particulier des portions où des arrêts de bus sont installés sur la rocade qui longe la muraille. En certains endroits, des trouées sauvages ou dues aux séismes et non reconstruites offrent, en plus des portes et passages officiels, des raccourcis entre les quartiers intramuros et ceux des arrondissements environnants. Ces raccourcis sont abondamment pratiqués, et offrent autant d'opportunité d'une halte à l'écart du flux.

Ces opportunités et ces usages éphémères peuvent parfois s'inscrire un peu différemment dans le temps et l'espace. En se dirigeant vers les derniers restes de la muraille maritime, au sud, on peut voir plusieurs petits salons de plein air cimentés à la hâte et offrant sièges et ébauches de sofa composés de pierres, gravats et béton. De la même façon, certains périboles intérieurs entre les murs principaux ouvrent sur des friches invisibles qui peuvent devenir de confortables endroits de détente. Ici, cependant, plusieurs situations se présentent. Dans certains cas, l'occupation du lieu pose problème : soit que des luttes d'appropriation s'y développent, soit que des usages moins calmes s'y déroulent.

Certains usages éphémères prennent en effet un caractère plus actif : libations, marquages de l'espace, sexualité, parcours sauvages, consommations illicites. Tandis que les premiers exemples évoqués sont caractérisés par le retrait vis-à-vis de la scène quotidienne, ceux-ci prennent un caractère plus transgressif.

Les libations nocturnes qui se déroulent sur la muraille à Istanbul attirent la réprobation : si l'usage sauvage et nocturne du site est, déjà, non autorisé, la consommation d'alcool, juste à côté de petits quartiers traditionnels au contrôle social étroit représente une double transgression, à la fois légale et morale. Pourtant, de petits groupes se réunissent à la tombée de la nuit, quand le temps le permet, allument parfois un feu, et s'installent pour quelques heures. En journée, ces libations préfèrent l'abri des périboles intérieurs. Ces hommes ne sont pas des agitateurs, évidemment. Certains d'entre eux sont parfois des chômeurs ou des résidents de l'intérieur de la muraille, mais beaucoup sont de simples habitants qui viennent s'encanailler de temps à autre pour échapper à la routine du quotidien. En dépit des craintes 
véhiculées sur ces pratiques et de l'aspect spectral de ces braseros crépusculaires, l'ambiance sur les lieux est plutôt détendue ${ }^{12}$, au moins jusqu'en début de soirée.

Ces usages s'inscrivent également dans le temps long de l'histoire de la muraille, qui a, depuis la période byzantine, accueilli sous ses murs, côté extérieur, les fêtes et les défoulements, inscrivant dans les représentations, à côté de l'image du coupegorge ou du repoussoir, celle du défouloir. Les tavernes du quartier Rom sédentarisé de Sulukule ont été fermées en 1992, et le quartier détruit récemment, mais tout le secteur conserve ce caractère ambigu. Ces micro-transgressions du quotidien peuvent aussi être solitaires : libations, onanisme. Les buveurs solitaires peuvent chercher de la compagnie à un certain point, et manifester de l'acrimonie vis-à-vis " du système ", de la vie en général, voire faire preuve d'agressivité si l'on souhaite mettre un terme à la rencontre.

De jeunes adultes se retrouvent pour flirter dans les endroits discrets de la muraille. Leur tenue vestimentaire, notamment pour les femmes, voilées, montre que ces envolées transgressent les codes moraux et religieux de leur quotidien. La sexualité est également présente sur la muraille, même si les derniers lieux de prostitution organisée ont disparu il y a trois ou quatre ans.

Ces pratiques transgressent les codes et les usages établis, sans pour autant prétendre changer la vie. Certains passages à l'acte permettent de compenser des frustrations nées du décalage entre les possibilités sociales et économiques réelles des personnes, et les appels explicites à des modes de vie différents qui s'étalent en grand sur les publicités, dans les rues et dans les médias. Les ressources financières et les codes moraux, familiaux, religieux interdisent une satisfaction directe de ces envies, qui trouvent un dérivatif ou une résolution dans les pratiques transgressives sur

$\overline{12}$ On apprend assez vite les dangers réels : d'abord les chiens sauvages qui hantent certaines portions, on peut aussi éviter le péribole qui jouxte la porte de Topkapi, puis les heures du cœur de la nuit à certains endroits (à Topkap1, près d'Edirnekap1, l'ancien cimetière arménien sous le Tekfur Saray), et puis bien sûr le fait de transgresser soi-même des appropriations stabilisées à l'intérieur de la muraille (habitats, certains terrains de maraîchers). 
la muraille. Mais surtout, au-delà des frustrations, ces pratiques permettent d'échapper un temps au contrôle social quotidien, et donnent aux usagers un sentiment de vivre, d'exister que l'on trouve bien dans l'excitation momentanée de ces pratiques. Il s'agit d'affirmer sa capacité à agir, à être. Certains incendies allumés par des enfants et jeunes adolescents à la crypte de Silivrikapı peuvent être compris aussi comme cela : au-delà de la provocation envers les adultes qui occupent discrètement les lieux à l'abri des regards, ces enfants n'ont pas tenté d'occuper euxmêmes le site (comme cela a pu être le cas ailleurs, par exemple au donjon d'Anemas).

Les deux types de pratiques, retrait et transgression, apparaissent ainsi comme deux modalités voisines de la différenciation. Le retrait renvoie à une forme de décompression, une volonté de s'extraire du flux quotidien, une pause ou un repos vis-à-vis du contexte normatif. Les transgressions, en revanche, expriment plus une volonté de s'affirmer, de se singulariser, une affirmation de soi en réaction à l'identification normative induite par l'affiliation. Le retrait renvoie à un équilibrage du poids normatif, tandis que la transgression, si elle est une manière plus vive de secouer le joug normatif, renvoie aussi à la construction de soi, au ressenti de soi. Les pratiques transgressives sont des moments où l'on peut se mettre à l'épreuve, où les individus veulent ressentir leur capacité propre à se singulariser en s'opposant.

Deux registres semblent engagés dans les usages éphémères : une modalité de relation de chaque usager au monde social, mais aussi, simultanément, une modalité de relation à soi-même. Passer d'une scène à l'autre engage non seulement la relation au monde mais aussi son identification : en changeant de contexte normatif, on choisit de se glisser dans un autre rôle, de jouer une autre partition, de se donner - aux autres et en même temps à soi-même - une autre image. L'investissement sur une scène secondaire peut être une ressource dans cette façon de se refaire le monde à soi-même, et s'objectiver dans des pratiques particulières de l'espace, qui visent d'abord à renégocier la vision que l'on a de sa position dans le monde. Dans ces différents cas, 
l'investissement en terme de secondarité, pour reprendre les termes de Jean Rémy, fonctionne de concert avec l'imaginaire, en tant que champ situé à la croisée des impulsions psychiques et sociales :

l'imaginaire traduit une ouverture vers un ailleurs qui, sans s'opposer systématiquement à l'ordre symbolique, rappelle néanmoins l'étendue des possibles. De ce fait, il permet de prendre distance face au symbolique. Dans sa poétique de l'espace, Gaston Bachelard explique que le drame des logements contemporains est l'absence de greniers. En les supprimant pour des raisons économiques, on a fait disparaitre un endroit privilégié où il était possible de se couper de la vie quotidienne en retrouvant les restes d'un monde sans ordre et révolu. Au grenier, on peut rêver d'être quelqu'un d'autre, on peut imaginer ce qui se serait passé si... ${ }^{13}$

L'enjeu serait alors l'existence possible de tels " greniers » à proximité des espaces-temps du quotidien et de la primarité...

La logique de l'écart s'objective donc de deux façons : par des pratiques de retrait et par des pratiques de transgression. Toutefois, le terme de transgression recouvre une variété de pratiques et de logiques. La transgression dont il est question ici est à distinguer, dans une certaine mesure, des transgressions qui visent à provoquer un changement dans l'ordre établi, de même que des transgressions codifiées relevant des rites de passage, ou encore de l'établissement de sociétés ou normes parallèles (banditisme, mafias, sociétés secrètes).

2.2. Transgression existentielle et transgression oppositionnelle

De la même façon que Marx différencie la révolte spontanée et la révolution organisée, il convient de distinguer les formes de micro-trangression spontanées et réactives, motivées par un besoin individuel d'exprimer son individualité, et qui n'ont pas pour but de changer la société, et les formes de transgressions plus conscientisées, orientées vers une mobilisation transformatrice, parfois porteuses d'un projet alternatif. S'il fallait proposer une première typologie, on pourrait distinguer des transgressions

13 Jean Rémy, Sociologie urbaine et rurale. L'espace et l'agir, Paris, L'Harmattan, 1998, p. 67-68. 
existentielles (du côté des logiques de l'écart) et des transgressions oppositionnelles, pour reprendre les termes d'Oskar $\mathrm{Negt}^{14}$.

Negt prend notamment pour point de départ les manifestations concrètes de l'opposition dans l'espace vécu. Il s'appuie sur un large registre historique, allant de la lutte contre le CPE en France en 2005 au mouvement ouvrier conseilliste du début du $\mathrm{XX}^{\mathrm{e}}$ siècle en Allemagne. Ces oppositions définissent un espace public oppositionnel, qu'il cherche à différencier de l'espace public bourgeois tel que défini au départ par Habermas, à qui il reproche d'oublier tous les moments de prise de parole et d'action des individus et des groupes qui ne peuvent prendre part au débat des savants et des notables. À travers ces mouvements et ces moments s'affirme une opposition concrète, dans laquelle s'expriment la conscientisation et le soulèvement contre un ordre établi, et qui peut déboucher sur une volonté de transformation sociale, d'organisation, de projet.

C'est en ce sens que l'on pourrait parler de transgressions oppositionnelles, pour qualifier les transgressions qui ont en commun une conscience et une volonté d'opposition à l'ordre établi. On peut penser par exemple aux procès récents contre les "Déboulonneurs ${ }^{15}$ " qui dégradent les publicités dans les rues et les transports urbains. Les occupations de site, les infiltrations dans des lieux interdits, les séquestrations sont encore des transgressions soutenues et motivées par une conscientisation et un projet plus ou moins défini.

Ce n'est pas le cas des transgressions existentielles, qui se situent à un niveau individuel, et effectuent un écart de conduite pour éprouver le sentiment d'exister, de se singulariser, sans envisager de transformer le contexte, et avec le désir ou la volonté de revenir au cours normal de la vie de tous les jours. Dans ces actes, on ne constate pas de prise de conscience collective, ni le passage par les étapes de la mobilisation et la montée en généralité des argumentations ${ }^{16}$.

14 Oskar Negt, L'Espace public oppositionnel, Paris, Payot, 2007.

15 Collectif français de lutte contre l'affichage publicitaire.

16 Programme de recherche d'initiative régionale "Mobilisations d'acteurs et gestion intégrée des espaces ", janvier 2006-octobre 2008, co-piloté par 
Pour autant, toutes les formes de transgression participent potentiellement à la transformation et à la reproduction de l'ordre social, sur des registres et des temporalités qui leur sont propres. Les transgressions existentielles peuvent avoir des effets matériels et collectifs : si aucune mobilisation collective ne semble envisageable, il est évident que leur concentration en certains points de la muraille définit pourtant une situation de recomposition socio-spatiale particulière, une identification par les différents acteurs, jusqu'aux autorités ou aux promoteurs parfois intéressés par l'exploitation du site. Par ailleurs, les individus, lorsqu'ils sont en action, peuvent passer d'une logique transgressive à une autre : ainsi être surpris inopinément par la police alors que l'on se défoulait simplement et sans intention précise peut faire basculer dans une opposition plus revendicatrice de liberté d'usage et de circulation. Enfin, certaines logiques peuvent se recouper : la transgression existentielle, qui consiste à se mettre en danger (pour soi ou devant quelques proches) afin de (se) prouver quelque chose, comme dans certains marquages de l'espace ou pénétration dans des lieux interdits, s'inscrit aussi dans des rites de passage qui peuvent être très ritualisés - et déboucher eux-mêmes dans certains cas sur des transgressions plus systématisées et conscientisées.

Aussi, la distinction entre transgressions existentielles et oppositionnelles a-t-elle une dimension analytique : elle définit deux bornes, qui ne se rencontrent pas toujours aussi radicalement dans les faits, et avec une pluralité de positions croisées. Mais elle est utile car elle permet d'aller plus loin dans la proposition analytique, et d'engager plus avant la discussion sur la dialectique homogénéisation-différenciation, en tentant de dépasser l'opposition binaire pour aller vers autre chose. Je vais faire référence ici à un schéma explicatif présenté une première fois en 2010 puis dans un texte publié cette année ${ }^{17}$, et dont les recherches qui

l'équipe ESO-Rennes de l'UMR 6590 ESO, et le Centre de Recherche sur l'Action Politique en Europe (CRAPE), CNRS-UMR 6051, IEP de Rennes, 2008.

17 Franck Dorso, «Le Pas de côté. Logiques de l'écart et régulations informelles dans l'espace urbain ", dans Manuel Boucher (dir), Regards croisés sur la 
ont eu lieu depuis et la réflexion engagée par cet article permettent d'enrichir la portée (cf. infra).

\subsection{Schéma pour-contre / avec-sans (PCAS)}

Les autorités publiques et les acteurs qui portent un discours légitime sur l'urbain (média, experts, promoteurs) regroupent souvent dans la même catégorie les émeutes urbaines, les mouvements revendicatifs qui transgressent la loi et les micro-transgressions dont nous venons de parler. Ces "dégradations " ou "déviances" sont identifiées comme des rebellions, l'expression d'une opposition à la normalité, à l'ordre établi, reconnu et légitimé. En cela on pourrait les classer dans un pôle " contre ", opposé à un pôle " pour », qui rassemblerait quant à lui tous les "bons comportements", conformes aux normes dominantes et à ce que l'on attend des individus. La programmation urbaine peut accompagner ce mouvement, en prévoyant dans les projets urbains les usages que feront les individus et les groupes des sites nouvellement aménagés. De la même façon, les lois sur les comportements (les rassemblements, ou les "dérives urbaines» dans la loi de sécurité intérieure de 2003 en France) ou le débat sur les signes extérieurs d'appartenance religieuse dans l'espace public montrent la vaste étendue du "pour » et les enjeux qui lui sont liés. Mais le plus souvent le " pour " est intériorisé par les acteurs ou agents sociaux, il s'agit simplement des normes dominantes de comportement et d'usage dans la vie sociale.

Les transgressions existentielles sont donc rangées dans la catégorie du " contre ", alors qu'elles relèvent à mon sens d'une toute autre logique. En fait, on peut regrouper le " pour» et le " contre " dans un ensemble, que l'on pourrait qualifier de " avec ", et mettre cet " avec " en tension avec un "sans ». Les bons comportements comme les usages oppositionnels prennent tous deux les normes à bras le corps, si l'on peut s'exprimer ainsi, dans un dialogue frontal ou conscientisé : on peut bien s'opposer à l'ordre existant, le fait de le faire montre bien qu'on le reconnaît

régulation des désordres, Paris, L'Harmattan, coll. « Recherche et transformation sociale ", 2012, p. 165-182. 
et que l'on converse - avec plus ou moins de rudesse - avec lui.

Â l'inverse, le « sans » désignerait les usages de l'écart (retrait et transgression existentielle) qui prennent un moment de la distance vis-à-vis du contexte normatif. Ils ne se situent pas dans un " hors-norme ", ou dans l'anomie, mais dans des champs normés un peu différemment, ce qui permet de ressentir un écart au contexte normatif vécu au quotidien.

Ainsi le schéma prend cette forme :
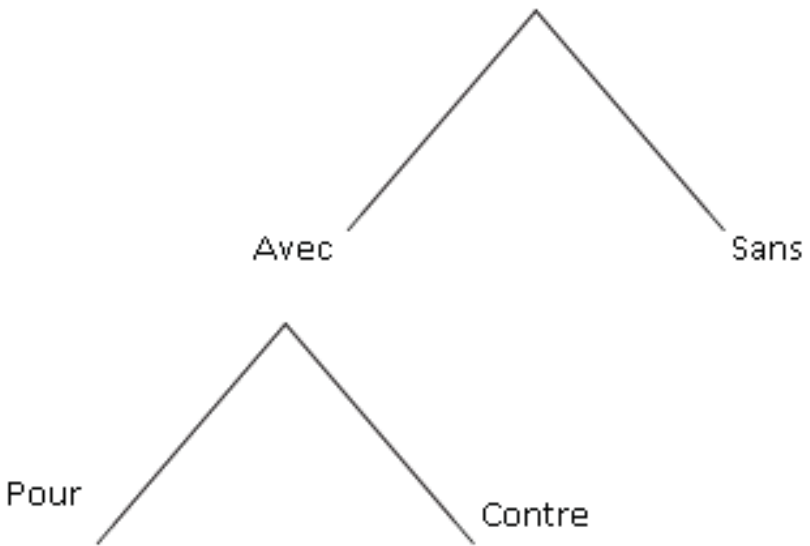

Ce schéma pour-contre / avec-sans (PCAS) est une proposition analytique en travail, elle évolue en fonction des recherches et des échanges - il faudrait d'ailleurs réfléchir au retour du " sans » vers le " pour " par son rôle de soupape et de régulation qui favorise la conservation et la reproduction du système. Mais l'apport que j'y vois maintenant, par les échanges engagés dans la cadre de la revue, et dans cette réflexion sur la tension homogénéisation-différenciation, est que ce schéma propose de sortir de la logique binaire (pour-contre, comme similitude-différence, ressemblance-dissemblance, affiliation-opposition), pour aller, non pas vers un logique ternaire, mais vers une double niveau de tension.

Le " sans " en effet n'est pas une troisième position, qui se situerait au même plan que le " pour " et le " contre " - et qui pourrait préfigurer, dans le champ de l'action, une version 
apaisée, un entre-deux, une solution. Il renvoie à un autre moment. "Pour " et "contre » forment en fait un même ensemble, en prise avec le champ normatif. Le " sans " n'appartient pas à ce champ, il représente les échappées, les sorties de ce champ. Elles lui restent liées, mais différemment : en tant que soupapes de décompressions nécessitant une extériorité, une sortie provisoire du champ.

Cette arborescence conserve donc les couples d'opposés structurants, mais propose de compléter cette architecture par un dédoublement vers un second niveau de tension, qui permet à son tour d'envisager un "sans ". Ce "sans " existe déjà sous certaines formes dans la recherche sociologique, de même que l'écart. Mais, dans cette articulation, il offre une perspective analytique à continuer d'explorer. Il constitue peut-être là, au moins dans le domaine de l'action urbaine, un point aveugle qui pose problème : peut-on imaginer les espaces du "sans ", ou mieux : peut-on imaginer qu' existent, sans intervention trop curieuse ni experte, des espaces du " sans " - dans la mesure où les logiques de l'écart ne peuvent se déployer que dans des cadres situés hors de la programmation et de l'institutionnalisation?

\section{Conclusion}

Les logiques de l'écart montrent une modalité particulière de la tension homogénéisation-différenciation, dans laquelle ce qui se présente spontanément comme une différence est en fait une condition de la reproduction sociale, de la similitude, de l'identité. Ce caractère paradoxal est double, car l'écart ne peut jouer ce rôle régulateur que si celui-ci demeure tacite et reste dans le non-dit. Une fois explicité, il ne peut plus jouer son rôle de soupape de décompression vis-à-vis du contexte normatif. Ce double paradoxe constitue une piste centrale pour avancer dans le projet d'une sociologie de l'écart.

D'autres pistes de travail peuvent être évoquées, à l'issue de cette réflexion sur l'écart et la différence. Il est d'abord nécessaire de situer l'écart dans la théorie sociologique, afin de préciser le sens qu'il peut revêtir ici. Il faut notamment comparer la 
démarche développée avec au moins trois conceptions de l'écart : l'écart comme élément perturbateur, l'écart comme équilibrage et l'écart dans sa dimension dialectique, c'est-à-dire la façon dont il interroge la place du binaire dans la vie sociale (outil analytique, structuration concrète dans les faits ?).

Sur le plan de l'analyse, on pourra également chercher à explorer l'amont et l'aval des processus présentés. En amont, il serait intéressant de remonter à la dynamique interne de la tension affiliation-différenciation qui innerve les logiques de l'écart : qu'est-ce qui fait qu'un individu ou un groupe va passer à l'acte, puis réintégrer le cours normal de l'existence ? Va-t-on vers une position d'équilibre entre affiliation et différenciation, ou vers une oscillation ? Y a-t-il un mouvement permanent ou un état latent et un état actif, autrement dit y a-t-il des écarts en permanence ou bien seulement sporadiques, à des moments éloignés les uns des autres? En aval, se posent les questions de résolution ou non de la tension : quels liens entre permanence et changement dans le modèle des tensions structurantes et des compromis successifs ? Que se passe-t-il quand surviennent des blocages qui interdisent les compromis transitoires? A-t-on alors un dévoilement, un affaiblissement, une transformation de la tension, des phénomènes d'anomie?

L'analyse doit dans le même temps se poursuivre sur le terrain. Il faudra en particulier continuer d'explorer les liens ambigus entre l'écart et la manière de concevoir, de gérer et de penser la ville. Dans la police de la cité, les usages informels ne sont vus souvent que dans leur dimension de différence, et bien moins dans leur participation (indispensable?) à l'existant et à sa reproduction. Il n'est pas sûr, on le pressent, que ce malentendu doive à tout prix être dissipé : peut-être constitue-t-il une ressource de l'écart pour pouvoir remplir sa fonction de soupape ?

Plusieurs domaines peuvent être explorés sur le terrain. D'abord l'évolution des rapports entre les institutions urbaines et les actes et acteurs de l'informel, en s'appuyant notamment sur la comparaison internationale. Ensuite en interrogeant la place possible des supports et lieux de l'écart dans l'évolution de l'action 
urbanistique, en particulier pour deux tendances qui, pour des raisons différentes, menacent l'existence de tels espaces-temps de pratiques : le renouvellement urbain et le développement sécuritaire. Enfin en explorant le rapport paradoxal de ces régulations informelles à la fois avec les politiques publiques sociales et avec les acteurs alternatifs qui mettent en avant une empathie spontanée mais parfois pleine de quiproquos avec ces usages et ces logiques - ceux-ci, loin de devenir des ferments de transformation sociale, sont plus souvent des garants de la conservation de l'ordre établi.

Les recherches qui se déroulent actuellement en Île de France, autour des usages des rives le long de la Marne et de la Seine et aux abords des infrastructures de transport montrent des convergences avec les résultats obtenus en Turquie, et sur d'autres terrains dans des villes moyennes en France. La comparaison pourra éclairer des similitudes mais aussi certainement des différences entre les processus de l'écart dans des contextes différents. Ce travail pourrait enfin ouvrir, dans le champ de l'action, non pas sur des transferts, car les relations entre l'écart et le champ institutionnel ne prennent pas la forme de protocoles, mais peut-être sur des idées ou des inspirations dans le regard sur les désordres, sans préjuger des apories éthiques et idéologiques que ne manquent pas de soulever ces désordres conservateurs.

\section{Bibliographie}

Agier, Michel, L'Invention de la ville. Banlieues, townships, invasions et favelas, Amsterdam, Éditions des archives contemporaines, 1999.

Blanc, Maurice, «La Transaction, un processus de production et d'apprentissage du "vivre-ensemble" ", dans Maurice Blanc, Marie-France Freynet et Gérard Pineau (dir.), Les Transactions aux frontières du social, Lyon, Chronique Sociale, 1998, p. 219-238. 
Dorso, Franck, "Le Pas de côté. Logiques de l'écart et régulations informelles dans l'espace urbain ", dans Manuel Boucher (dir.), Regards croisés sur la régulation des désordres, Paris, L’Harmattan, coll. " Recherche et transformation sociale ", 2012, p. 165-182.

Dorso, Franck, "Informel », dans L'Abc de l'urbanisme, Créteil, Université Paris-Est Créteil éditeur, 2010, p. 91-94.

Dorso, Franck, La Part d'ombre. Transactions et conflits entre les usages informels et les opérations de rénovation de la muraille de Théodose II à Istanbul, mémoire de thèse, Strasbourg, CRESS EA 1334, Université Marc Bloch, 2008.

Girard, Muriel, Recomposition du monde artisanal et mutations urbaines au regard des mises en patrimoine et en tourisme au Maghreb et au MoyenOrient (Fès, Istanbul, Alep), mémoire de thèse, Tours, UMR 1835 CITERES, Université François Rabelais, 2010.

Goffman, Erwin, La Mise en scène de la vie quotidienne, tome 2, Les relations en public, Paris, Minuit, 1973.

Ledrut, Raymond, L'Espace en question, Paris, Anthropos, 1976.

Monnet, Jérôme, «Dissociation et imbrication du formel et de l'informel : une matrice coloniale américaine ", Espaces et sociétés, 2010, vol. 3, n 143, 2010, p. 13-29.

Negt, Oskar, L'Espace public oppositionnel, Paris, Payot, 2007.

Pérouse, Jean-François, La Turquie en marche, Paris, La Martinière, 2004.

Programme de recherche d'initiative régionale, Mobilisations d'acteurs et gestion intégrée des espaces, janvier 2006-octobre 2008, co-piloté par l'équipe ESO-Rennes de l'UMR 6590 ESO et le Centre de Recherche sur l'Action Politique en Europe (CRAPE), CNRS-UMR 6051, IEP de Rennes, 2008.

Rémy, Jean, Sociologie urbaine et rurale. L'espace et l'agir, Paris, L'Harmattan, 1998.

Rémy, Jean et Liliane Voyé, Ville, ordre et violence, Paris, PUF, 1981.

Rémy, Jean, Liliane Voyé et Émile Servais, Produire ou reproduire, tome 1, Conflits et transactions sociales, Bruxelles, Vie ouvrière, 1978.

Simmel, Georg, Sociologie et épistémologie, Paris, Presses universitaires de France, coll. "Sociologies », [1894-1912] 1991. 